\title{
Phenatrene biodestructors isolated from soils of large cities
}

\author{
Grigorii Kozlov ${ }^{1}$, Mikhail Pushkarev ${ }^{1, *}$, and Viktoria Mokhna ${ }^{1}$ \\ ${ }^{1}$ Saint-Petersburg State Institute of Technology, 190013, 26 Moskovsky prospect, St. Petersburg, \\ Russia
}

\begin{abstract}
The article shows the possibility of bacterial degradation of crystalline phenanthrene in its pure form. The results make it possible to create a technology for processing chemical industry waste in the form of "as is" without dilution with neutral substrates or clean soils. Bacteria Pseudomonas sp., Completely destroying crystals moistened with a solution of salts of Raymond's medium, were isolated from a soil sample taken in Soho Square, London (UK). Similar destructors, but with less activity, were isolated from the soil of the industrial zone of Dzerzhinsk (Russia).
\end{abstract}

\section{Introduction}

The development of bioremediation technologies today has made it possible to relatively cheaply clean landscapes contaminated with oil products and polycyclic aromatic hydrocarbons, but we always deal with dispersed contaminants, or are forced to dilute the waste with clean substrates. Attempts to solve the problem are being undertaken - it is proposed to use instead of clean substrates, in particular, sludge from sewage treatment plants, the article [1] presents the results of studies on the use of sludge from sewage sludge as a substrate for composting together with PAHs. The method gives good results on biodegradation of pollutants, and can also be used in winter due to self-heating of the composted mass, however, the thermophilic composting phase ends on the 20th day, when the degree of destruction of PAHs is $50-60 \%$, it is also necessary to add to the compostable mass structuring materials (straw, sawdust). The use of composting contaminated soils with the application of fertilizers for cleaning soils contaminated with oil products, including benz [a] pyrene at low temperatures in northern Sakhalin, is described. The average rate of oil decomposition was $157 \mathrm{mg} / \mathrm{kg}$ of soil per day, the duration of soil cleaning was 95 days [2]. The use of compost from MSW for biodegradation of PAHs [3] also does not solve the actual problem of destruction of concentrated waste, but only offers a substrate for their dilution.

In this regard, it is necessary to search for new strains of destructors in order to search for strains highly destructive to pollutants - destructors.

\footnotetext{
*Corresponding author:malexpush@bk.ru
} 


\section{Materials and methods}

To search for PAH destructors, soil images were selected in large cities of Russia and the world (St. Petersburg, Moscow, London, Los Angeles, San Francisco, New York, Madrid) using the "passive expedition" method - samples were taken and delivered to the laboratory by our request as an accompanying task on business trips or visits. From each point, 3 replicates were selected. Soil and soil sampling was carried out in sterile paper bags, then delivered to the laboratory. The work also used soil samples from Russian industrial cities with a high level of pollution.

The selection of microorganisms was carried out on selective liquid storage media. A sample of soil weighing $1 \mathrm{~g}$ was sterilely placed in a $750 \mathrm{ml}$ Erlenmeyer flask with $100 \mathrm{ml}$ of Raymond mineral medium, phenanthrene phenanthrene was added as the only carbon and energy source at a concentration of $0.1 \%$, it was kept on a rocking chair with a rotation speed of $220 \mathrm{rpm}$, at temperature $25^{\circ} \mathrm{C}$. The cultivation period was 7 days. After the first passage, $10 \mathrm{ml}$ of liquid were taken and sterilely introduced into a new medium with a phenanthrene concentration of $1 \%$. Put on a rocking chair at $220 \mathrm{rpm}$ for 7 days. Each week, $10 \mathrm{ml}$ were collected and subcultured on fresh medium. After 4 weeks, the cultures were planted on solid nutrient media with phenanthrene applied to the surface.

The study of the properties of cultures was carried out using classical microbiological culture media, dyes and techniques. Identification was also carried out using the MALDITOF mass spectrometry method based on the Scientific Research Institute named after Pasteur (St. Petersburg). For mass spectrometric studies, a small number of microorganisms were selected from Petri dishes and applied to the target. In parallel, the same number of microorganisms were applied to the adjacent matrix well and covered with a lysis buffer consisting of $70 \%$ formic acid solution, $30 \%$ acetonitrile solution and $0.1 \%$ sodium dodecyl sulfate (SDS) solution for better destruction of the cell walls and allowed to dry. After the sample dried, $1 \mu \mathrm{l}$ of the matrix ( $\alpha$-cyano-4-hydroxycinnamic acid) was layered on it. Mass spectrometric identification of microorganisms was performed on a MicroFlex mass spectrometer (Bruker, Germany). Each sample was tested in duplicate. Spectra were taken in automatic mode. Standard detection mode - MBT_FC (linear polarization, with a detection range of proteins from 2-20 kDa). 240 spectra were obtained from each sample, which ultimately overlap each other. Identification was performed using the MALDI BioTyper database (version 3.0; if [MSP] 5627 cell microorganisms) (Bruker, Germany). To determine the degree of agreement of the proposed method with the reference, bioinformatic statistics was used - a measure of agreement, which has a maximum of 3.00 with full agreement, and is from 0 to 1,699 if the agreement between the estimates (tests) is observed no more often than might be expected by coincidence, when both indications from 1,700 to 1,999 show approximate results. Values from 2,000 to 2,299 are considered a good degree of agreement, which allows you to accurately determine the type of culture, but the genus of culture does not have a $100 \%$ guarantee of identification. Coefficients from 2,299 to 3,000 show $100 \%$ coincidence of the genus and species of microorganisms.

\section{Results and discussion}

The natural reservoir of microorganisms-destructors of organic ecotoxicants in general and polycyclic aromatic hydrocarbons in particular are natural biocenoses with a high level of anthropogenic pollution, such as large cities and territories of chemical industry enterprises. The spectrum of microorganisms capable of destroying PAHs is very wide [4]; therefore, the released destructors, as a rule, are not of significant interest. In addition, the growth of most of these destructors is possible only with insignificant concentrations of harmful 
substances, and for effective degradation it is often necessary to use auxiliary technological methods, for example, vermiculture [5] or phytoremediation [6].

In the search for promising PAH destructors in three samples, we discovered microorganisms that can destroy pure phenanthrene moistened with the mineral base of Raymond's nutrient medium. Cultures were isolated from an accumulative culture with soil samples from Soho Square in London (Great Britain), Figure 1, and Dzerzhinsk (Russia).

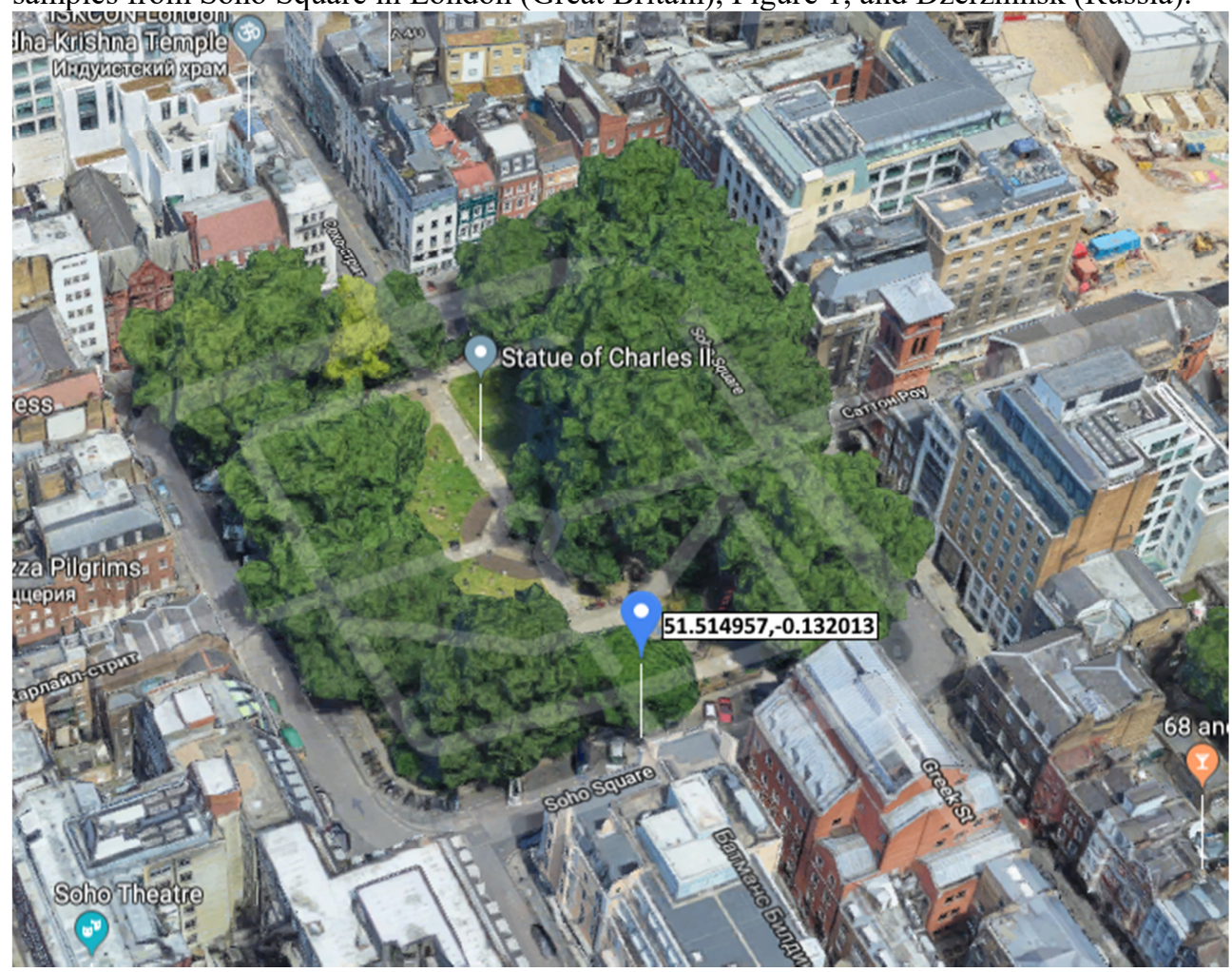

Fig. 1. Place of sampling, from which a culture is selected that performs the most active utilization of crystalline phenanthrene

A distinctive feature of destructors capable of destroying crystals is the formation of pigment during cultivation, both on liquid (Figure 2 and 4) and on solid nutrient media (Figure 3 and 5). Cultures with no pigment did not show the ability to destroy crystalline phenanthrene. In the accumulation cultures shown in Figures 1 and 3, pigmentation is very different, which clearly correlates with the results of the decomposition of phenanthrene when plated on solid nutrient media. In all cases, only pigment-containing bacteria can degrade crystalline phenanthrene.

This property does not appear on naphthalene, anthracene, acenaphthene, acenaphthylene and persists only when the association is re-sown on media with phenanthrene; the use of culture media with other carbon sources leads to the loss of this property. 


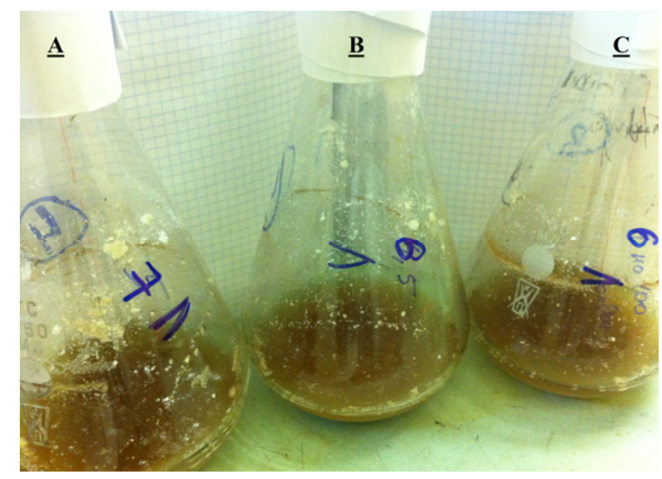

Fig. 2. Cumulative culture with soil samples taken in Soho Square, London.

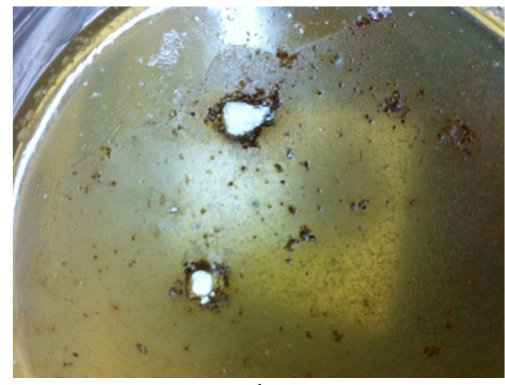

1

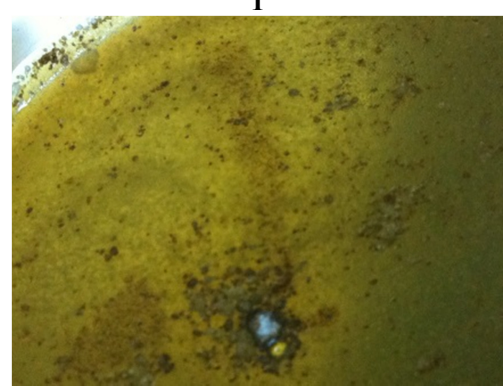

3

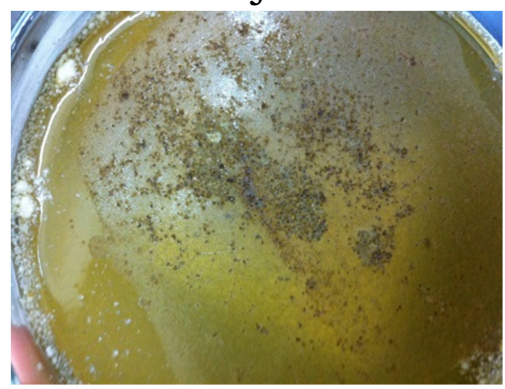

5

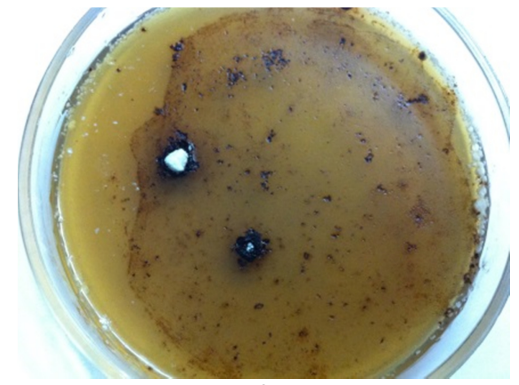

2

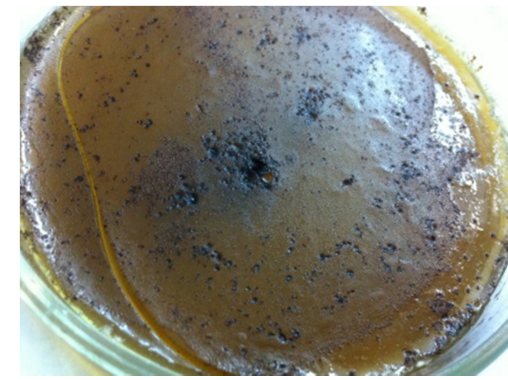

4

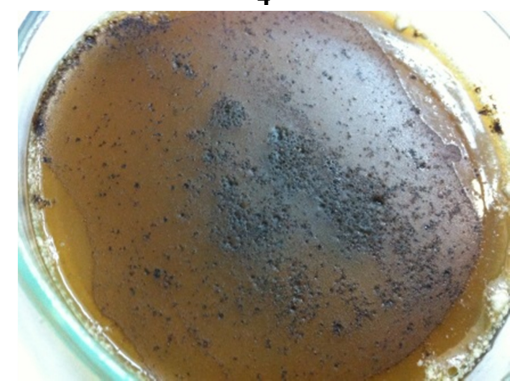

6

Culture A: 1 - 14 days, 2 - 30 days. Culture: B - 3-14 days, 4-30 days.

Culture: $\mathrm{C}-5-14$ days, 6-30 days.

Fig. 3. Biodegradation of crystalline phenanthrene on the surface of a dense nutrient medium by microorganisms of an accumulative culture with soil samples taken in Soho Square, London. 


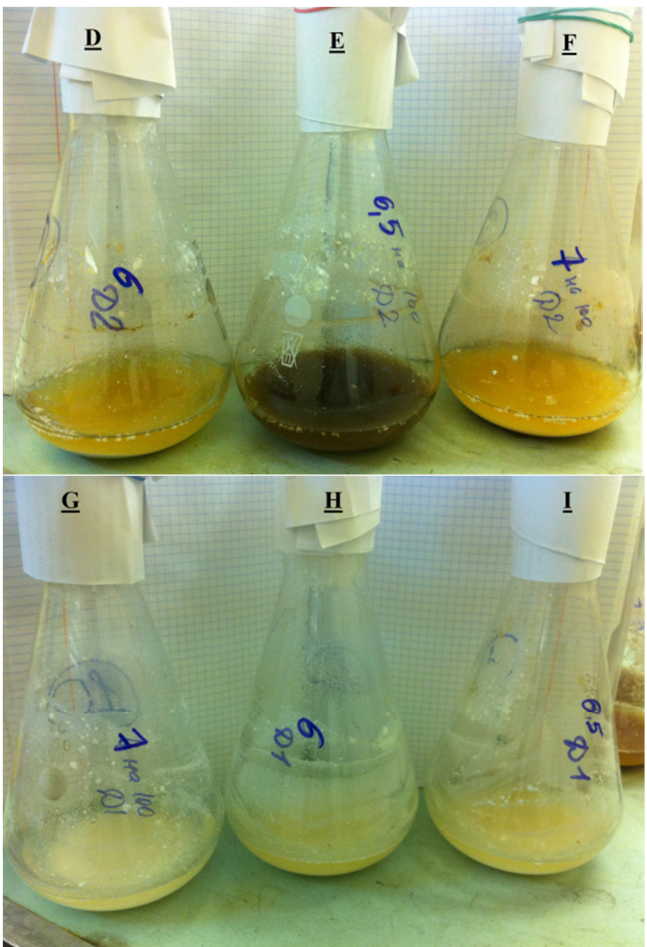

Figure 4. Cumulative culture with soil samples taken in the industrial zone of Dzerzhinsk.

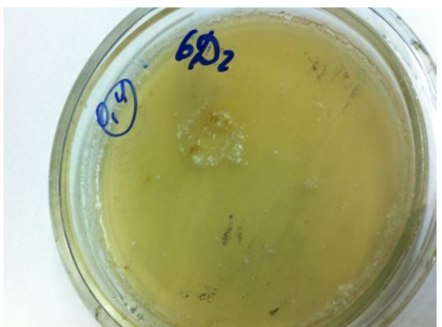

1

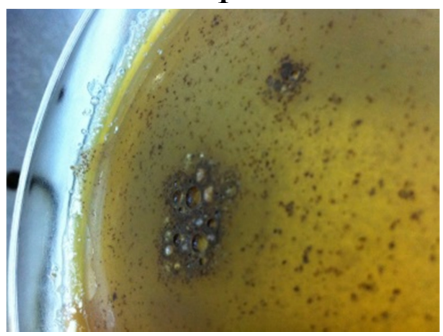

3

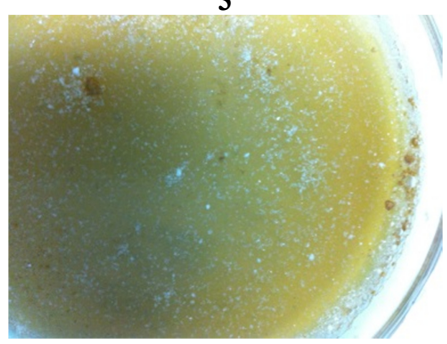

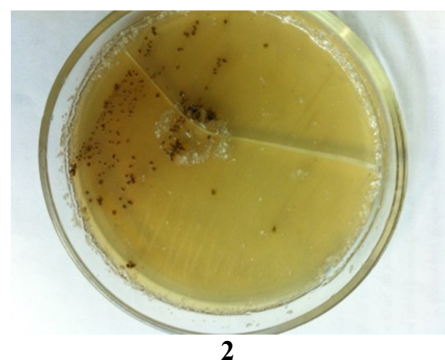

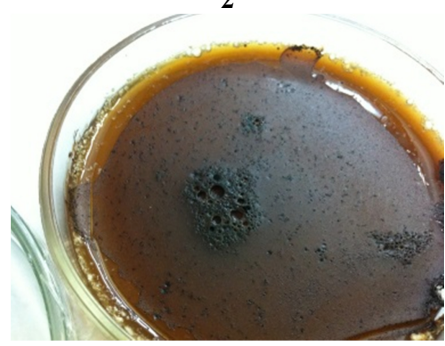

4

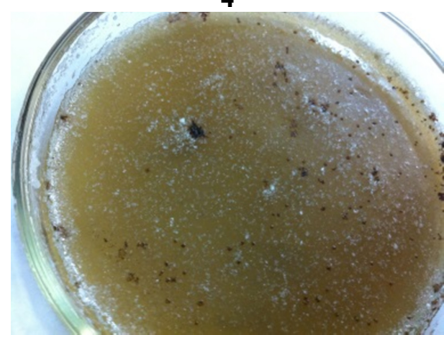


Culture E: 1 - 14 days, 2 - 30 days. Culture: F - 3 - 14 days, 4 - 30 days. Culture: I - 5-14 days, 6-30 days.

Fig. 5. Biodegradation of crystalline phenanthrene on the surface of a dense nutrient medium by microorganisms of an accumulative culture with soil samples taken in the industrial zone of Dzerzhinsk.

A culture isolated from the soil of London showed the highest resistance to high concentrations of phenanthrene (Table 1), showing that the soil of cities with a long industrial history is a very promising ecological niche for searching for strains of PAH destructors. It was this culture that was selected for further study.

Table 1. Growth of crops at various concentrations of phenanthrene

\begin{tabular}{|c|c|c|c|}
\hline $\begin{array}{c}\text { Phenanthrene } \\
\text { concentration }\end{array}$ & $\begin{array}{c}\text { Dzerzhinsk (culture I } \\
*)\end{array}$ & $\begin{array}{c}\text { Dzerzhinsk (culture E } \\
* \text { ) }\end{array}$ & London (culture B *) \\
\hline 0,1 & + & + & + \\
\hline 0,5 & + & + & + \\
\hline 1,0 & - & + & + \\
\hline 1,5 & - & +- & + \\
\hline 2,0 & - & - & + \\
\hline 2,5 & - & - & + \\
\hline
\end{tabular}

* Designation of crops according to figures 1 and 3.

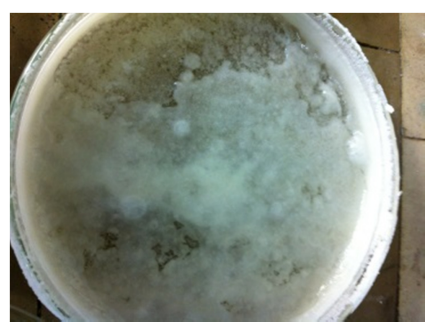

1

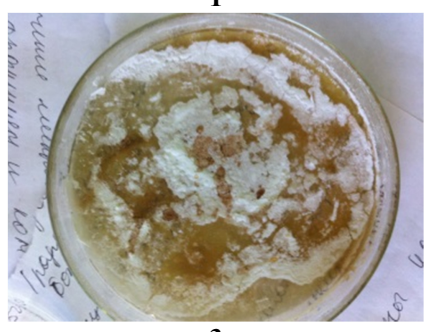

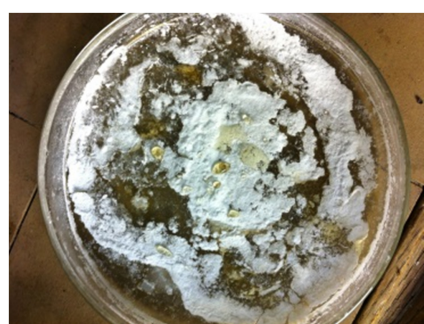

2

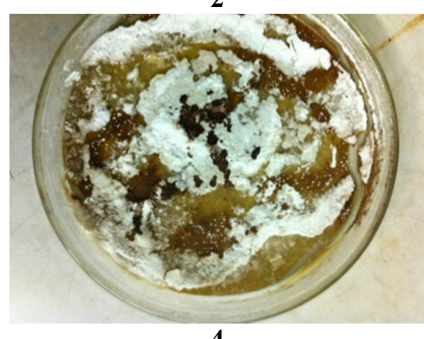

B: 1 - initial medium; 14 days, 2 to 10 days; 3 to 20 days, 4 to 30 days.

Fig. 6. Biodegradation of crystalline phenanthrene on the surface of a dense nutrient medium in a layer of $1 \mathrm{~mm}$ by microorganisms of an accumulative culture

The next step was to study the possibility of biodegradation of phenanthrene not in the form of separate crystals and a thin film on the surface of the medium, but in the form of a layer of powder. Raymond agar medium was prepared, on the surface of which phenanthrene was applied with a layer of 1 and $4 \mathrm{~mm}$. Culture growth was noted only in the variant with a layer thickness of $1 \mathrm{~mm}$, with a layer thickness of $4 \mathrm{~mm}$, there was no growth. At the same time, pigmentation of bacterial colonies - destructors was also observed (Figure 6). 
According to the results of the analysis of cultural-morphological characters and MALDI-TOF mass spectrometry, the culture was identified as Pseudomonas sp.

\section{Conclusions}

1. The soils of large cities with a long industrial history are promising natural reservoirs for the isolation of microorganisms-destructors of ecotoxicants.

2. Biodegradation of PAH (phenanthrene) wastes in the form of pure substances without dilution with neutral substrates is possible, however, the layer thickness at which the effect was observed was $1 \mathrm{~mm}$, which makes further selection of the strain necessary.

\section{References}

1. L. Hua, W. Wu, Y. Liu, Y. Chen, M. B. McBride, Water, air, and soil pollution 193(14), 259-267 (2008).

2. G. P. Golodyaev, N. M. Kostenkov, V. I Oznobikhin, Eurasian Soil Science 42(8), 926-935 (2009).

3. M. Farrell, D. L. Jones, Bioresource technology, 100(19), 4301-4310 (2009).

4. H. Lee, S. Y. Yun, S. Jang, G. H. Kim, J. J. Kim, Bioremediation Journal 19(1), 1-8 (2015).

5. S. B. Chachina, N. A. Voronkova, G. V. Kozlov, M. A. Gross, O. N. Baklanova, AIP Conference Proceedings 2141, 020002 (2019); https://doi.org/10.1063/1.5122021

6. T. Spriggs, M. K. Banks, P. Schwab, Phytoremediation of polycyclic aromatic hydrocarbons in manufactured gas plant-impacted soil. Journal of environmental quality, 34(5), 1755-1762 (2005). doi:10.2134/jeq2004.0399 